\title{
On Spatial Reuse and Capture in Ad Hoc Networks
}

\author{
Naveen Santhapuri \\ University of South Carolina \\ Email: santhapu@cse.sc.edu
}

\author{
Srihari Nelakuditi \\ University of South Carolina \\ Email: srihari@cse.sc.edu
}

\author{
Romit Roy Choudhury \\ Duke University \\ Email: romit@ee.duke.edu
}

\begin{abstract}
Neighbors of both the transmitter and the receiver must keep quiet in a 802.11 wireless network as it requires bidirectional exchange, i.e., nodes reverse their roles as transmitters and receivers, for transmitting a single DATA frame. To reduce role reversals and to improve spatial reuse, a piggybacked acknowledgment based approach has been proposed to enable concurrent transmissions. Recent findings on physical layer capture show that it is possible to capture a frame of interest in the presence of concurrent interference and that the SINR threshold is dependent on the relative order in which the frame and the interference arrive at the receiver. In this paper, we show that it is possible to exploit capture and increase concurrent transmissions in wireless adhoc networks. We develop a distributed channel access scheme and demonstrate that it offers significant throughput gain particularly at lower data rates.
\end{abstract}

\section{INTRODUCTION}

Achieving optimal network capacity for wireless networks in the presence of interference is a challenging task and it is fundamentally related to spatial reuse. Efficient spatial reuse is inhibited by interference, limitations of MAC protocols, external noise and many other physical factors. The 802.11 protocol with its virtual carrier sensing has role reversals which reduce the hidden node problem but introduce the exposed node problem, further restricting spatial reuse.

Multiple packets arriving at a receiver are generally considered to cause packet loss due to the collision at the receiver. For this reason, nodes in a wireless network avoid transmitting concurrently to mitigate interference at the expense of spatial reuse. However, there have been several studies that have shown that a sufficiently stronger frame can still be successfully received by the receiver in spite of a collision [1] [2]. This phenomenon is called physical layer capture (PLC).

If we approach the concurrent transmission problem with the knowledge of this interesting effect, there is scope for improvement although the role reversals are still a hurdle. In this paper, we propose a MAC protocol which reduces role reversals and takes advantage of the PLC to improve the number of concurrent transmissions in wireless adhoc networks. Our MAC protocol makes use of the channel condition information obtained by the physical layer in making a good assessment of the channel and staggers transmissions to achieve concurrency.

The rest of the paper is organized as follows. Section II provides the background, details of the capture model and the method for reducing role reversals. Section III describes the proposed capture-aware MAC protocol in detail. Section IV presents the results of simulations in QualNet evaluating the performance of our protocol. We compare our work with other related works in section V before concluding in section VI.

\section{BACKGROUND}

Role Reversals: 802.11 networks counter the ill affects of the hidden terminal problem by using physical carrier sensing and the 4-phase (RTS-CTS-DATA-ACK) MAC protocol. In this protocol, each node reverses its role (transmitter to receiver and vice versa) twice for delivering one DATA frame. With role reversals, all nodes around the transmitter and receiver will cautiously remain silent even if they do not affect the reception. This is called the exposed node problem which greatly reduces the spatial reuse in 802.11 networks. Several schemes like [3] have been suggested to alleviate this problem by making optimizations to the MAC protocols. These protocols address the exposed node problem to some extent, but the primary condition that the SINR value be above a high threshold seriously limits the possibilities. Two neighbors cannot transmit simultaneously unless the SINR value at each of their receivers is greater than a high threshold.

Alleviating Role Reversals If there was no ACK phase in the protocol, two nodes can transmit DATA simultaneously without worrying about the reception of ACK and the exposed sender problem can be solved partially. But, the ACK phase is the only way a node can know about the success/failure of a transmitted DATA packet. We proposed a remedy for this problem using a piggybacked ACK mechanism in [4] and we use the same mechanism in this work. The piggybacked ACK mechanism encapsulates acknowledgements for multiple neighbors in each of the packets (RTS, CTS, DATA) transmitted by a node. Eliminating the ACK phase reduces one role reversal and removes one hurdle for concurrency.

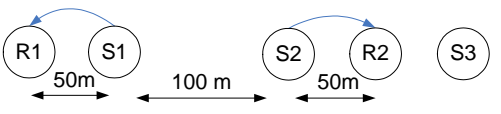

Fig. 1. Concurrent transmissions possible due to physical layer capture effect even when all nodes are within the range $(357 \mathrm{~m}$ for $12 \mathrm{Mbps})$ of each other.

Capture Effect: The phenomenon of physical layer capture was characterized in [1] by experimentation. The authors demonstrated that a stronger frame can be received correctly even if it starts after the beginning of an interfering frame. An example of capture corresponding to Fig. 1 is shown in Fig. 2. In this sample topology, R1 can receive a packet from $\mathrm{S} 1$ in spite of the interference from S3, even if the packet from 

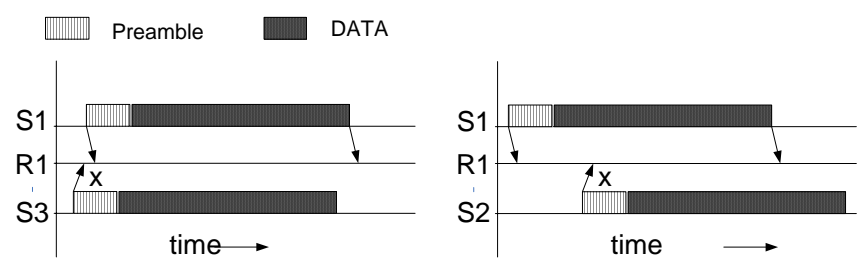

Fig. 2. (a) During the plcp reception phase a stronger frame arrives from $\mathrm{S} 1$ at $\mathrm{R} 1$ while it is receiving a frame from S3. R1 captures the frame from $\mathrm{S} 1$ because it is much stronger than the frame from S3; (b) When the frame from $\mathrm{S} 1$ is past the plcp phase, an interfering frame arrives at R1 from S2. Node R1 will filter the interference from S2 (though it is stronger than the inference from $\mathrm{S} 3$ ) and continue to receive the signal from $\mathrm{S} 1$.

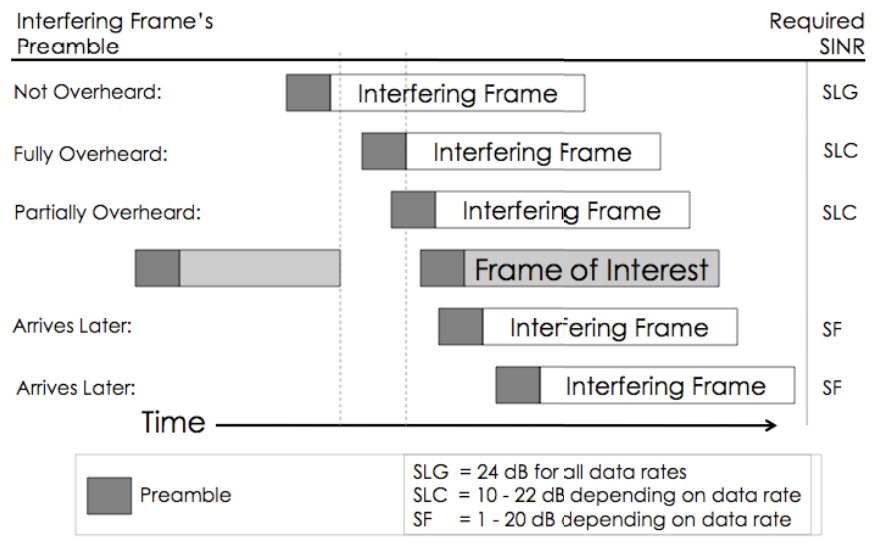

Fig. 3. The order of frame arrivals and its relation to SINR threshold: i) If interfering frame arrives later, SINR threshold is the least (SF); ii) If frame of interest arrives in the presence of known interference, the SINR threshold is medium (SLC); iii) If frame of interest arrives in the presence of unknown interference, SINR threshold is the highest (SLG).

$\mathrm{S} 1$ arrives later at $\mathrm{R} 1$ than the packet from S3. The recent work in [2] provides the clearest picture yet, of the capture phenomenon and the various SINR thresholds dictated by the timing of the signal and the interference. From these two works we can conclude that a signal is significantly more vulnerable to interference if it starts after the interfering frame than had it started before the interfering frame.

The effect of the timing of signal and interference arrivals is shown in Fig. 3 which is based on the model in [2] which uses message-in-message (MIM) mode. There are various SINR thresholds instead of the single value commonly used in literature. We use the same terminology (SF, SLC, SLG) for these thresholds as in [2]. If a frame arrives in the absence of any interference in the sensitivity region, the SINR threshold is lower and is called SF (Sender First). If an interfering frame arrives at the receiver, and if the receiver hears the plcp (physical layer preamble) of the interfering frame, the frame of interest which arrives later is subject to a higher SLC (Sender Last Clear) threshold. If an interfering frame arrives at the receiver earlier, but its plcp cannot be understood, the frame of interest will be subject to the highest threshold SLG (Sender Last Garbled). SF and SLC increase with data rate and SLG is reported to be almost same for all data rates. This variation in threshold values is a major factor in determining the extent of concurrency feasible in a wireless network. If the MAC layer is aware of this aspect of the capture, protocols can be designed to exploit capture and improve spatial reuse.

\section{OUR APPROACH}

\section{A. Advantage of Capture Awareness}

We first list the requirements for concurrency in 802.11 and show how they can be relaxed with capture-awareness.

Signal strength conditions for 802.11: Assume $S_{i j}^{k}$ is the SIR (Signal to Interference Ratio) value at $j$ for a signal from $i$ in the presence of interference from k. Given two transmitterreceiver pairs (S1-R1, S2-R2), the following conditions must hold for concurrent DATA transmissions to happen in 802.11:

1) Both pairs must be completely out of range of each other

2) The following SINR values must be > SLC: $S_{S_{1} R_{1}}^{S_{2}}$, $S_{R_{2} S_{2}}^{S_{1}}, S_{S_{2} R_{2}}^{S_{1}}, S_{S_{1} R_{1}}^{R_{2}}, S_{S_{2} R_{2}}^{R_{1}}, S_{R 2 S_{2}}^{R_{1}}, S_{R_{1} S_{1}}^{S_{2}}, S_{R_{1} S_{1}}^{R_{2}}$

These conditions ensure that the RTS, CTS, DATA and ACK can be received without any errors caused by interference.

Capture Aware Staggered Transmissions: The conditions for concurrent transmissions are less stringent when taking PLC into account because a captured packet requires a lesser SINR threshold than a packet arriving in the presence of interference, for most data rates. We can stagger the transmissions to satisfy the SINR requirements and achieve more concurrency. We call our approach capture-aware staggering of transmissions (CAST) and illustrate below.

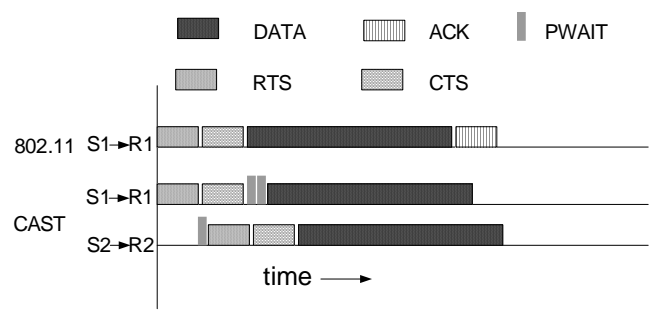

Fig. 4. Concurrency possibilities with capture. By waiting 2 PWAIT times, the first transmission allows the second one to take place concurrently

We use the QualNet physical propagation model (which takes the higher value of the free-space and plane-earth models for path-loss) with 802.11 a $12 \mathrm{Mbps}$ data rate for this illustration. The transmission power is $19 \mathrm{dBm}$ and the range is approximately 350 meters. Consider the topology in Fig. 1 where S1 and S2 send packets to R1 and R2 respectively. Suppose the following signal strength conditions hold:

1) Each value in $\left(S_{S_{1} R_{1}}^{S_{2}}, \quad S_{R_{2} S_{2}}^{S_{1}}, \quad S_{S_{2} R_{2}}^{S_{1}}, \quad S_{S_{1} R_{1}}^{R_{2}}\right.$, $\left.S_{S_{2} R_{2}}^{S_{1}}, S_{R 2 S_{2}}^{R_{1}}, S_{R_{1} S_{1}}^{S_{2}}, S_{R_{1} S_{1}}^{R_{1}}\right)>\mathrm{SF}$

2) Each value in $\left(S_{S_{2} R_{2}}^{R_{1}}, S_{S_{1} R_{1}}^{R_{2}}, S_{S_{2} R_{2}}^{S_{1}}, S_{R 2 S_{2}}^{R_{1}}\right)>$ SLC

We can then have two concurrent transmissions as shown in Fig. 4 by ordering the transmissions as follows:

1) RTS: $S 1 \rightarrow R 1$, no other frames so SINR $>$ SF holds.

2) CTS: $R 1 \rightarrow S 1$ and RTS: $S 2 \rightarrow R 2$ after one physical preamble wait time. When the CTS starts, the medium is free and hence SF holds. Once RTS starts, the CTS frame is past the capture phase and $S_{R_{1} S_{1}}^{S_{2}}>\mathrm{SF}$. 


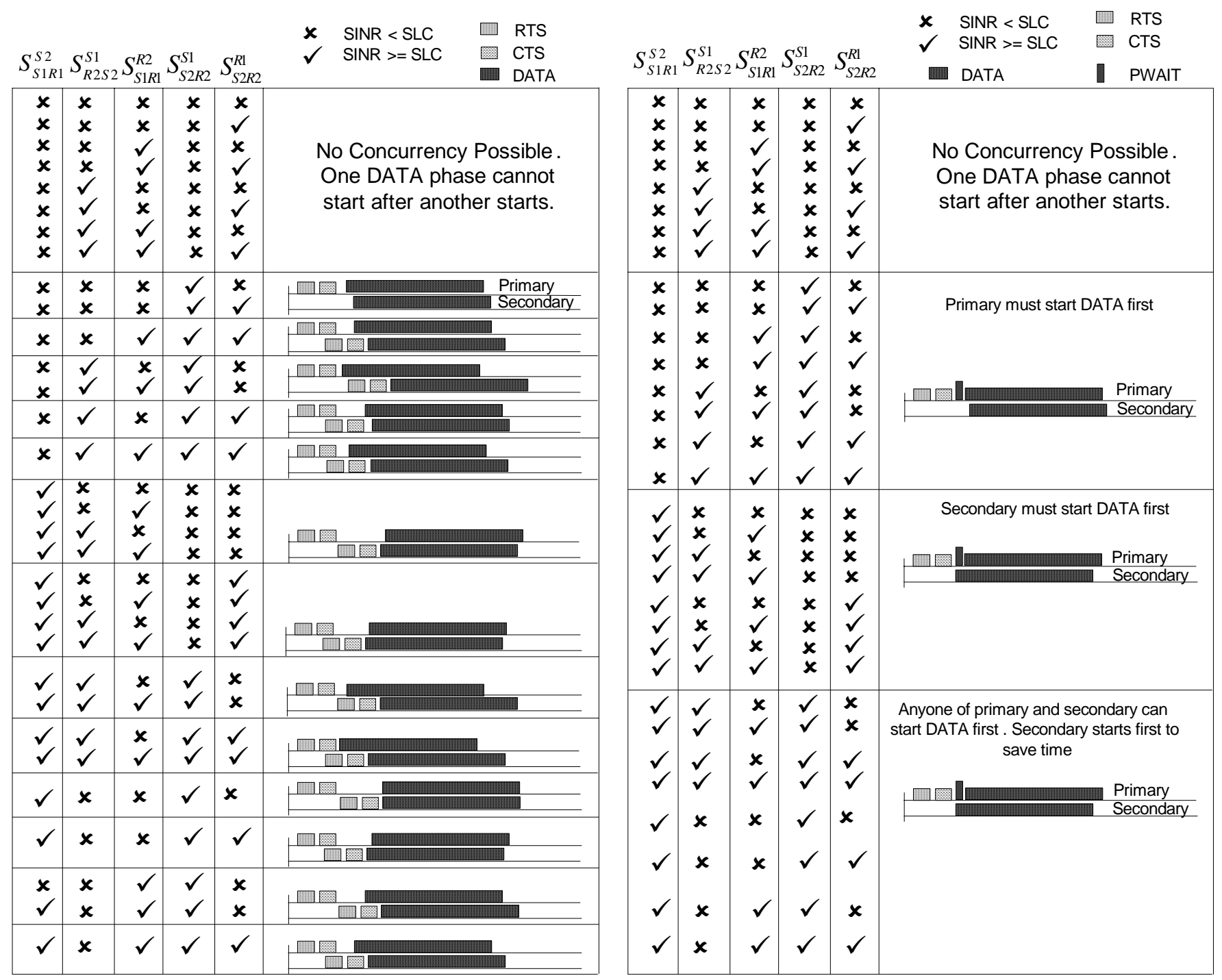

(a) Concurrency Possibilities with capture and staggering: Combinations 1-8 (b) Concurrency with just DATA as secondary transmission. Helps realize cannot have any concurrency because $S_{S_{1} R_{1}}^{S_{2}}$ and $S_{S_{2} R_{2}}^{S_{1}}$ both fail and hence the full potential of staggering and PLC without significant changes. Which if one DATA phase starts first, the other cannot start. Rest of the combinations transmitter enters the DATA phase first depends on the values $S_{S_{1} R_{1}}^{S_{2}}$ and show the possible staggering in protocol phases to take advantage of capture $S_{S_{2} R_{2}}^{S_{1}}$
and achieve concurrency.

Fig. 5. Staggering the Phases of Primary and Secondary Transmissions for Concurrency

Therefore S1 can continue receiving CTS. Since $S_{S_{2} R_{2}}^{R_{1}}$ $>$ SLC, R2 can start receiving RTS frame.

3) CTS: $R 2 \rightarrow S 2$ and DATA: $S 1 \rightarrow R 1$ after 2 physical preamble times. The CTS will start when the medium is free and therefore SF holds. The DATA frame starts after the capture phase of CTS frame and since $S_{S_{1} R_{1}}^{R_{2}}$ $>$ SLC both frames can continue to be received.

4) DATA $S 1 \rightarrow R 1$ and DATA: $S 2 \rightarrow R 2$. Since $S_{S_{1} R_{1}}^{S_{2}}>$ SF (DATA frame past the capture phase) and $S_{S_{2} R_{2}}^{S_{1}}>$ SLC both DATA frames can be received.

The idea is to make the primary transmitter let a concurrent transmitter take advantage of the PLC wherever possible. The primary transmitter is made to wait for 2 PWAIT (physical preamble time) times for the secondary to enter its CTS phase so that the SF value becomes the required SINR threshold at the secondary transmitter to receive the CTS.

\section{B. Concurrency Possibilities with Staggering}

As mentioned above, there are eight signal strength values which must be above SLC threshold for two concurrent transmissions to take place under 802.11. Assume SF threshold condition holds for all of them, the RTS phase of primary is over and the CTS phase has started. If we are using piggybacked ACKs, we only need to consider 5 signal strengths $\left(S_{S_{1} R_{1}}^{S_{2}}, S_{R_{2} S_{2}}^{S_{1}}, S_{S_{2} R_{2}}^{R_{1}}, S_{S_{1} R_{1}}^{R_{2}}, S_{S_{2} R_{2}}^{S_{1}}\right)$ and each of them have to be greater than SLC. Fig. 5(a) shows how staggering and PLC can be used together to achieve concurrency when different combinations of these conditions 
are true. By staggering the RTS, CTS and DATA phases of both pairs of nodes appropriately to satisfy the lower SINR thresholds, we can achieve concurrency wherever it is possible. Fig. 5(a) enumerates what is feasible. But realistically, it is hard to implement a different kind of staggering for each case without significantly altering the basic working of the MAC protocol. Below we present a more practical alternative.

We can optimize the protocol by making the secondary transmission send only data without RTS-CTS ${ }^{1}$. Fig. 5(b) shows the possibilities with DATA as secondary transmission. It can be seen that this protocol will require just one of the two signal conditions $\left(S_{S_{1} R_{1}}^{S_{2}}>\mathrm{SLC}\right.$ or $\left.S_{S_{2} R_{2}}^{S_{1}}>\mathrm{SLC}\right)$ to be true. In general, for any concurrency to occur, at least one of those two conditions must be true because a secondary data transmission cannot start if SLC doesn't hold during the capture phase. If the secondary just has DATA: 24 out of 32 combinations can have concurrency whereas with 802.11 , only 1 out of 32 possibilities can have concurrency. This change to the protocol increases the opportunities for concurrency due to fewer constraints on SINR thresholds. The overhead (maximum 2 PWAIT times) is negligible even at the highest data rates because the ACK phase is removed.

\section{Scheme}

1) Assumptions: We assume two hop signal strength information (i.e., S1's signal strength at R1 will be known by S2) for this protocol. This information can be obtained by making each node create and broadcast a list of average signal strength values of its neighbors. Several schemes to calculate link interference were proposed in [5] and [6].

2) MAC Protocol Decision at Secondary Transmitter: Without the RTS-CTS for secondary transmissions, we are able to relax most of the constraints to achieve concurrency. To transmit concurrently in an adhoc multihop wireless network, a node must be able to determine if a concurrent transmission is possible after hearing a RTS from a neighbor. If the primary waits for 1 preamble time before the DATA phase as shown in Fig. 5(b) there can be 4 possibilities for the secondary:

- $S_{S_{1} R_{1}}^{S_{2}}<$ SLC and $S_{S_{2} R_{2}}^{S_{1}}<$ SLC: S2 will not transmit because a concurrent transmission is not possible.

- $S_{S_{1} R_{1}}^{S_{2}}>\mathrm{SF}$ and $S_{S_{2} R_{2}}^{S_{1}}>$ SLC: In this, frame from $\mathrm{S} 1$ is the more vulnerable one. S2 waits for a preamble time after S1 starts the DATA phase. This lets S1 take advantage of the lower threshold by virtue of starting first.

- $S_{S_{1} R_{1}}^{S_{2}}>\mathrm{SLC}$ and $S_{S_{2} R_{2}}^{S_{1}}>\mathrm{SF}: \mathrm{S} 2$ starts transmitting before S1 starts its DATA phase. This helps R2 hear the physical preamble before S1 DATA phase starts and requires only $\mathrm{SF}$ threshold for R2's reception. Since $S_{S_{1} R_{1}}^{S_{2}}>$ SLC, S1's DATA can be received by R1 in the presence of interference from $\mathrm{S} 2$.

- $S_{S_{1} R_{1}}^{S_{2}}>$ SLC and $S_{S_{2} R_{2}}^{S_{1}}>$ SLC: Concurrent transmission is possible regardless of the order of DATA transmissions. We let S2 start DATA first to save time.

\footnotetext{
${ }^{1}$ The use of piggybacked ACKs makes this secondary transmission similar to DATA-ACK, which is quite common in 802.11 networks.
}

3) Multiple Secondary Transmitters: If a secondary transmitter is unaware of another secondary transmission, the multiple interference may cause collisions at all the receivers. For this reason we allow concurrency only when a neighbor is the transmitter (in other words only when a secondary hears the RTS from the primary). To avoid 2 transmitters starting at the same time, each secondary will have a small contention window (size 5) and will pick a slot in the contention window randomly. If a secondary detects additional interference before transmitting the packet, it will abort the transmission assuming that some other secondary has started transmission. We understand that there will be signal strength variations which might cover up any increase in interference and hence the avoidance of secondary transmissions is not certain in reality. Our heuristic to estimate interference (described below) at the receivers will help in avoiding most of the multiple interference effects. Each slot is $4 \mu$ s and will give sufficient time for a node picking the next slot to hear the signal. This will increase the wait time between CTS and DATA of primary by 5 slot times $(20 \mu \mathrm{s})$. So instead of waiting for 1 preamble time the primary waits for preamble time $+20 \mu \mathrm{s}$.

4) Estimating Interference at the Receivers: When a secondary transmitter S2, makes a decision based on the SINR at $\mathrm{R} 1$, it must take into account external noise and other possible interferences to reflect the actual SINR value. Since the only information $\mathrm{S} 2$ has is the $S_{S_{1} R_{1}}^{S_{2}}$ value, it must estimate the noise and interference at both the receivers based on the noise and interference in its vicinity. Instead of using a complex estimation, we use a simple heuristic in our scheme. We always make a conservative estimate by assuming that the interference and noise at the receiver is higher by a cushion factor than that at the transmitter. We ran the simulations with different cushion factors and found the results to be similar. Here, we show performance gains with 1.1 as cushion factor.

\section{EVALUATION}

We implemented an SINR-threshold based physical layer capture model that is described in [2] and our MAC protocol along with the piggybacked ACK mechanism in the QualNet [7] simulator. We had to modify the carrier sensing mechanism to let the nodes transmit concurrently when a neighbor is transmitting. Since we use a piggybacked ACK, our protocol uses a delayed backoff mechanism to compensate for a packet loss because there is no explicit ACK. Our simulation consists of two phases. In the first phase the 2 hop signal strength information is exchanged. In the second phase the actual traffic simulation is conducted.

\section{A. Collecting Signal Strength Information}

In the first phase, the physical layer gathers and passes the signal strength information of all neighboring nodes to the MAC layer. The MAC layer embeds the signal strength information of all of its neighbors and broadcasts a HELO packet. This way the two hop neighbors will receive the corresponding signal strength information. Nodes take turns to 

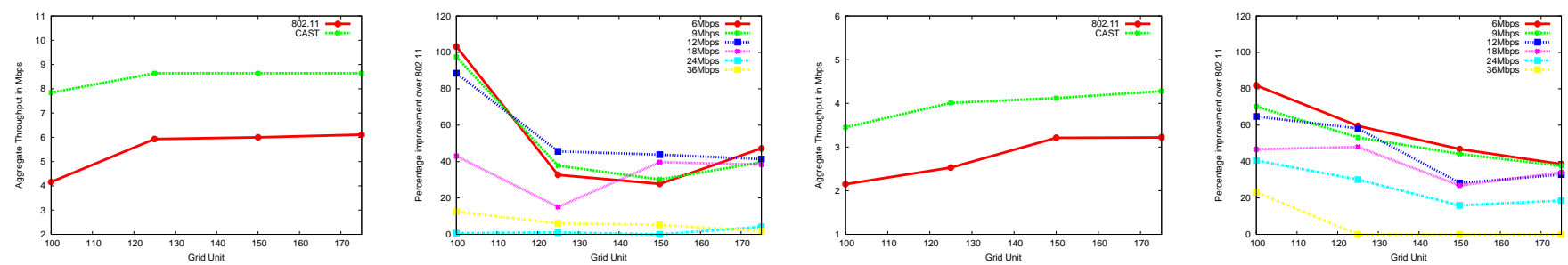

Fig. 6. $5 \times 5$ Grid : (a) 2 hop flows (i) throughput for $12 \mathrm{Mbps}$ and (ii) gains for all rates; (b) 3hop flows (i) throughput for $12 \mathrm{Mbps}$ and (ii) gains for all rates.
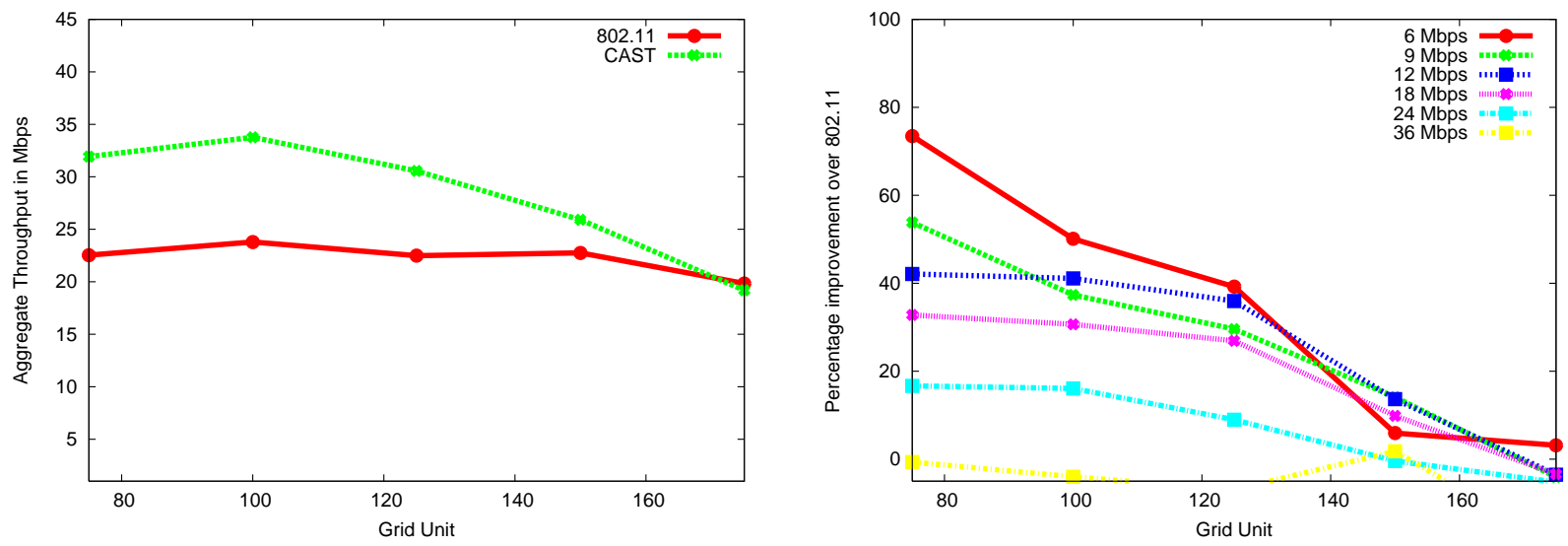

Fig. 7. Grid topologies in a fixed size area: (a) aggregate throughput for 12Mbps; (b) percentage improvements for various data rates.

disseminate this information and all HELO packets are sent at the lowest data rate to ensure reliable and long range delivery.

\section{B. Topology and Traffic Simulation}

The traffic flows in all the scenarios except the small grid are generated randomly and the number of flows is sufficient to saturate the network. Each of the flows is a CBR flow with 512 byte sized packets. The number of packets per second is greater than required for saturation at the corresponding data rate. We used static routing in all cases. We compared our MAC protocol + PLC model with the 802.11a model in QualNet at various data rates for the following 3 topologies.

Small Grid: Our basic evaluation was with the $5 \times 5$ grid topology using the same set of 2- and 3-hop flows (4 flows in each set) as in [8]. We present the throughput comparison for $12 \mathrm{Mbps}$ and percentage improvements for all data rates in Fig. 6 for 2-hop flows and 3-hop flows.

Grids in a fixed sized area: We performed simulations on several grid topologies in a 1000x1000 m space. Each grid has a different grid unit (ranging from 75 to $175 \mathrm{~m}$ ) and as many nodes as possible in the available space. Fig. 7 shows the results of this evaluation setting.

Random Topologies: We also performed simulations in a random topology of 100 nodes in a 1000×1000 m area. We randomly generated 40 1-hop flows with hop distances constrained to a maximum value. The simulations are repeated with different set of flows for varying max hop distance. The evaluation results are shown in Fig. 8.

All the results show consistent improvement across different scenarios which help us arrive at the following conclusions:
- Long hop distances require higher SINR thresholds for signal reception and therefore the scope for improvement is less. Shorter hop distances yield the highest improvements for the same reason.

- The improvements are higher at lower data rates, because the difference between SF and SLC thresholds becomes lesser and lesser as data rates increase, and consequently the scope for improvement over 802.11 decreases.

- The aggregate throughput can be significantly improved depending on the hop distance and the data rate.

\section{Higher Data rates}

We did not consider data rates over $36 \mathrm{Mbps}$. At rates greater than $36 \mathrm{Mbps}$, the distance between interferer and receiver must be 10 to 15 times more than the distance between transmitter and receiver to satisfy the SF threshold (20-22dB). Given a carrier sense range of $350 \mathrm{~m}$ and requirement that concurrent transmitters be in range of each other, this higher SINR threshold drastically reduces the scope for improvement ${ }^{2}$.

\section{RELATED WORK}

Several works studied the spatial reuse problem and various solutions involving power control, carrier sense and MAC protocol tuning have been proposed. The exposed terminal problem is addressed in [3] by allowing multiple pairs of nodes complete the RTS/CTS phase before everyone transmits DATA such that the ACK phases are synchronized. In [9]

\footnotetext{
${ }^{2}$ Even though the data rate caps have increased (from $1 \mathrm{Mbps}$ in 802.11 to about $128 \mathrm{Mbps}$ in $802.11 \mathrm{n}$ ), there is still a necessity for transmissions at lower data rates, due to the high bit error rates at higher data rate transmissions.
} 

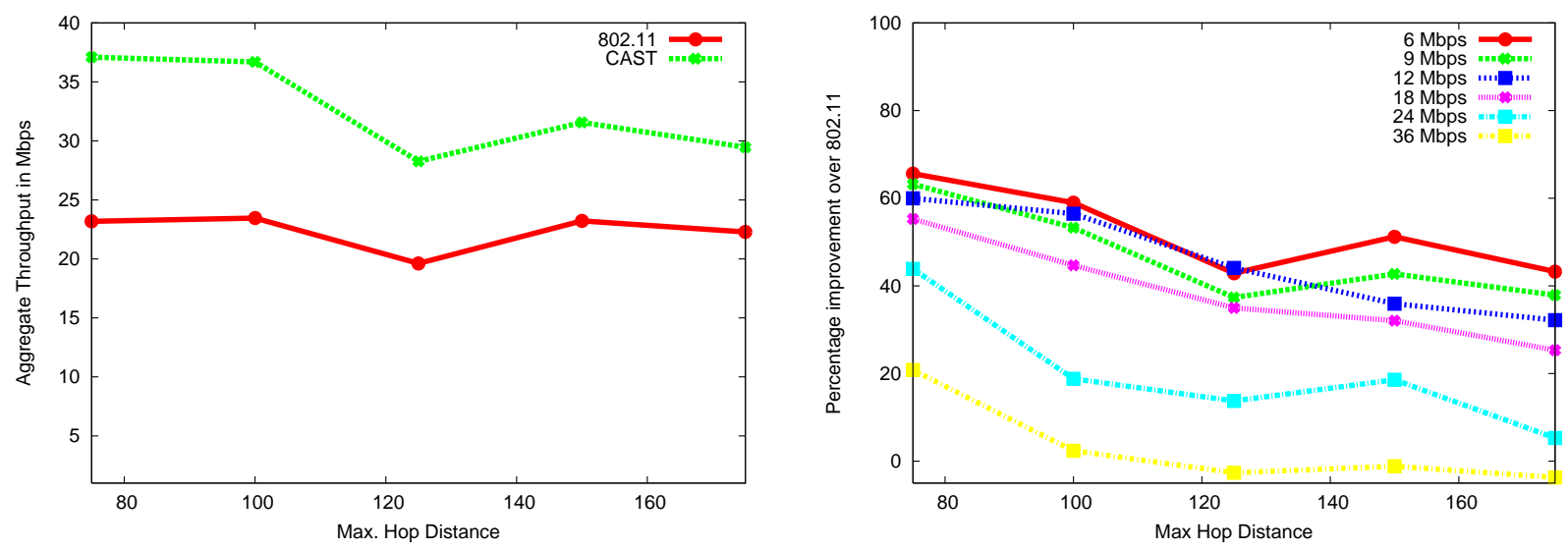

Fig. 8. Random topology with 1-hop flows with varying max hop distance: (a) aggregate throughput for 12Mbps; (b) percentage gain for all data rates.

the authors allow a secondary DATA-only transmission to take place if it is smaller than the primary DATA. In [8], nodes distributedly decide when to transmit simultaneously by making use of the received signal strength metric and the RTSS/CTSS messages. This approach is interesting but it does not take capture into account. In [10] the authors propose a centralized power and rate control algorithm to improve spatial reuse. In [11] the authors study the effect of carrier sensing and power control and conclude that a product of both should be a predetermined constant to achieve optimal spatial reuse. The use of piggybacked ACK instead of the explicit 802.11 ACK phase was proposed for reducing role reversals [4] and for improving throughput [12].

Many theoretical models like [13] have been proposed to explain physical layer capture. The first empirical evidence of capture we know of is [1] which defined the packet timing conditions for capture. The recent study in [2] quantifies the SINR threshold requirements for 802.11a networks under different packet arrival timings and gives a clear picture of this phenomenon. A similar work for low power wireless networks was done in [14]. Capture awareness has been used for collision resolution in [15]. In [16], the authors propose tuning the carrier sense threshold and show that there is scope for improvement if nodes are capture aware. The unfairness caused by capture is discussed in [17] and BER models for capture were proposed in [18]. In [19], a scheme is proposed to perform suitable beam forming and avoid 'capture' of packets by directional antennas in their idle state. This capture refers to locking on to an arriving signal and is different from the capture effect discussed in our current work. An $\mathrm{O}\left(n^{2}\right)$ algorithm for estimating link state interference in multihop wireless networks was proposed in [5] and a linear order algorithm that takes capture into account was presented in [6].

\section{CONClusions AND Future WORK}

Spatial reuse in wireless networks is limited by the SINR threshold requirements. This problem is amplified because of role reversals in wireless networks. Physical layer capture can improve the spatial reuse by staggering the transmissions. In this work we explored the possibilities by combining reduced role reversals with capture. Our simulation results show that the number of concurrent transmissions can be improved significantly though the scope for improvement reduces with the higher data rates for which the SINR requirements are very high. Our ongoing work includes further evaluation of the protocol and to develop distributed and centralized protocols for improving the performance of fixed wireless networks.

\section{REFERENCES}

[1] A. Kochut, A. Vasan, A. U. Shankar, and A. Agrawala, "Sniffing out the correct physical layer capture model in 802.11b," in ICNP, Oct. 2004.

[2] J. Lee et al, "An experimental study on the capture effect in 802.11a networks," in WinTECH, Sept. 2007.

[3] A. Acharya et al, "MACA-P: A MAC protocol to improve parallelism in multi-hop wireless networks," in PERCOM, 2003.

[4] N. Santhapuri, J. Wang, Z. Zhong, and S. Nelakuditi, "PiggybackedAck-aided Concurrent Transmissions," in ICNP Poster Session, 2005.

[5] J. Padhye et al, "Estimation of link interference in static multi-hop wireless networks," in $I M C, 2005$.

[6] J. Lee et al, "Rss-based carrier sensing and interference estimation in 802.11 wireless networks," in SECON, 2007.

[7] "Qualnet Network Simulator," http://www.scalable-networks.com/.

[8] K. Mittal and E. M. Belding, "Rtss/ctss: Mitigation of exposed terminals in static 802.11-based mesh networks," in WiMesh, Sept. 2006.

[9] D. Shukla, L. Chandran-Wadia, and S. Iyer, "Mitigating the exposed node problem in ieee 802.11 ad hoc networks," in ICCCN, Oct 2003.

[10] T-S Kim, H. Lim, and J. Hou, "Improving spatial reuse through tuning transmit power, carrier sense threshold, and data rate in multihop wireless networks," in Proc. ACM Mobicom, 2006.

[11] J. Fuemmeler et al, "Selecting transmit powers and carrier sense thresholds for csma protocols," in UIUC TechReport, Oct. 2004.

[12] R. R. Choudhury, A. Chakravarty, and T. Ueda, "Implicit mac acknowledgment: An improvement to 802.11," in 4th IEEE/ACM Wireless Telecommunications Symposium, Apr. 2005.

[13] O. Dousse M. Durvy and P. Thiran, "Modeling the 802.11 protocol under different capture and sensing capabilities," in Proc. IEEE Infocom, 2007.

[14] D. Son, B. Krishnamachari, and J. Heidemann, "Concurrent packet transmissions in low-power wireless networks," in SENSYS, 2006.

[15] K. Whitehouse et al, "Exploiting the capture effect for collision detection and recovery," in Emnets, May 2005.

[16] K. Jamieson et al, "Understanding the real world performance of carrier sense," in ACM SIGCOMM E-WIND Workshop, 2005.

[17] C. Ware, J. Chicharo, and T. Wysocki, "Unfainess and capture behavior in 802.11 adhoc networks," in ICC, June 2000.

[18] H. Chang et al, "A general model and analysis of physical layer capture in 802.11 networks," in Proc. IEEE Infocom, 2006.

[19] R. R. Choudhury and N. Vaidya, "Mac-layer capture: A problem in wireless mesh networks using beamforming antennas," in SECON, 2007. 